\title{
Possible altitudinal, latitudinal and directional dependence of relativistic Sagnac effect in Chern-Simons modified gravity
}

\author{
Daiki Kikuchi, Naoya Omoto, Kei Yamada, and Hideki Asada \\ Faculty of Science and Technology, \\ Hirosaki University, Hirosaki 036-8561, Japan
}

(Dated: June 25, 2021)

\begin{abstract}
Toward a test of parity violation in a gravity theory, possible effects of Chern-Simons (CS) gravity on an interferometer have been recently discussed. Continuing work initiated in an earlier publication [Okawara, Yamada and Asada, Phys. Rev. Lett. 109, 231101 (2012)], we study possible altitudinal and directional dependence of relativistic Sagnac effect in CS modified gravity. We compare the CS effects on Sagnac interferometers with the general relativistic Lense-Thirring (LT) effects. Numerical calculations show that the eastbound Sagnac interferometer might be preferred for testing CS separately, because LT effects on this interferometer cancel out. The size of the phase shift induced in the CS model might have an oscillatory dependence also on the altitude of the interferometer through the CS mass parameter $m_{C S}$. Therefore, the international space station site as well as a ground-based experiment is also discussed.
\end{abstract}

PACS numbers: 04.25.Nx, 04.50.-h, 04.80.Cc 


\section{INTRODUCTION}

Modifications of the theory of general relativity (GR) have been of interest. Particularly, some modifications that introduce second (or higher) order terms of curvature tensors represent high-energy corrections to the Einstein-Hilbert action. The Chern-Simons (CS) correction is one of modified gravity models. The CS modification is not an ad hoc extension, but it is actually motivated by both string theory, as a necessary anomaly-canceling term to conserve unitarity [1], and loop quantum gravity (LQG), as a counter term for the anomaly[2] and recently as the emergence of the CS gravity when the Barbero-Immirzi parameter of LQG is promoted to a scalar field and the Holst action is coupled to fermions [3]. CS modifications to gravity were first formulated in $2+1$ dimensions [4]. Several authors investigated the structure of these theories in $3+1$ dimensions to show that they could arise as a low-energy limit of string theory [5]. The theory and formulation of CS modified gravity have been discussed in a number of papers (see [6] for a review), and possible imprint of such a modification in the early universe has been recently investigated. Moreover, there has been little work on tests of such CS corrections in the present Universe.

In nondynamical CS gravity, a scalar field is assumed to be externally prescribed. It is often taken to be a linear function of the coordinate time (as a canonical choice), and induces parity violation in the theory. Nondynamical CS gravity depends on a single free parameter [7-9]. The constraint on this parameter with measurements of frame-dragging of bodies orbiting the Earth has been discussed; The proposal has been implemented by Ali-Haimoud and Chen [10] to constrain CS gravity; Yunes and Spergel, and Ali-Haimoud [11, 12] have used double-binary-pulsar measurements.

In addition to cosmological and astrophysical tests, current attempts to probe general relativistic effects in quantum mechanics focus on precision measurements of phase shifts in quantum interferometers (e.g. [13]). Toward a test of parity violation in a gravity theory beyond GR, Okawara and his collaborators have recently studied a possible constraint by neutron interferometers [14, 15], where they used Alexander and Yunes (AY) model. The main purpose of the present paper is to improve the previous results on the interferometers regarding two points $[14,15]$. One improvement is that the present paper considers an updated nondynamical CS model that has been developed by Smith, Erickcek, Caldwell, and

Kamionkowski (SECK) [9] in order to study both interior and exterior gravitational fields 
by a spinning object, whereas AY model assumes a point-like spinning object. SECK model can treat an extended source of the gravitational field and, in some limit, it approaches AY model. Because of including a mass parameter (through a homogeneous solution to the field equation), SECK model shows oscillating behavior of the gravitational potential along the radial direction of a central object. As a result, we shall study altitudinal dependence of Sagnac effect in the present paper. The other improvement is that we consider Sagnac interferometers in optics. This is more advantageous at present, mainly because it is relatively easy to put at different places Sagnac interferometers compared with neutron interferometers that need nuclear reactors as a source of neutrons.

This paper is organized as follows. Sec. II briefly reviews SECK model of nondynamical CS gravity theory and the relativistic Sagnac effect. In Sec. III, we compute relativistic Sagnac effects in the CS model. Sec. IV provides numerical calculations. Sec. V is devoted to conclusion.

Throughout this paper, Latin indices run from 1 to 3, while Greek ones from 0 to 3.

\section{RELATIVISTIC SAGNAC EFFECT AND NONDYNAMICAL CS MODIFIED GRAVITY}

This section summarizes the basics of computing a phase difference in Sagnac interferometer by CS modified gravity.

\section{A. Relativistic Sagnac effect}

The Sagnac effect, which is often called Sagnac interference, originally describes a phenomenon encountered in interferometry that is elicited by rotation. It appears manifestly in a setup called a ring interferometer. Similar effects due to relativistic gravitomagnetic fields in a stationary spacetime are often called relativistic Sagnac effects. For instance, see [16, 17] for a review of ring-laser tests of fundamental physics. Yet, a recent proposal of an experimantal scheme to measure the Lense-Thirring (LT) effect with a Sagnac interfer-

ometer is still a long way from reality (See e.g. [18]). Accroding to [18], G in Geodetic Observatory Wettzell, the best ring laser in the world, already achieves the accuracy within one order of magnitude from the expected signal for detections of LT effects. Aiming at the 
LT detection, they are now planning a new experiment called GINGER in order to reduce various sources of noises. Therefore, such a drastic experimental progress is a major premise for our ambitious proposal of using a Sagnac interferometer in order to testify to the CS effect separately from the LT one (that has not been detected with any interferometer yet).

Consider two beams of monochromatic light in a closed path (denoted by $C$ ) such as a ring or a square, where one beam is clockwise and the other anticlockwise. Along the light path, we have $d s^{2}=g_{\mu \nu} d x^{\mu} d x^{\nu}=0$. In the gravitomagnetic field, the leading contribution to the arrival time shift $\Delta t$ is given by the relativistic version of Sagnac effect as [19]

$$
c \Delta t=-2 \oint_{C} \frac{g_{0 i}}{g_{00}} d x^{i}
$$

where $C$ denotes a clockwise closed path of a light beam. This formula is almost the same as that for a matter wave [14, 15] except for a factor 2, if de Broglie wavelength is replaced by the photon wavelength. See [20,21] for relativistic higher order corrections. Dividing the time shift by the wavelength of a photon $\lambda$, we obtain the phase difference as

$$
\Delta \Phi=\frac{2 \pi}{\lambda} c \Delta t
$$

Let us consider experiments near the surface of Earth, for which we can assume a small perturbation around the Minkowskian background spacetime as $g_{\mu \nu}=\eta_{\mu \nu}+h_{\mu \nu}$. The timespace component of the metric does matter in the relativistic Sagnac formula. It is denoted as a spatial vector $\vec{h} \equiv\left(h_{01}, h_{02}, h_{03}\right)$. The leading order of Eq. (1) becomes [19]

$$
c \Delta t=-2 \int_{S}(\vec{\nabla} \times \vec{h}) \cdot d \vec{S}+O\left(h^{2}\right),
$$

where we used Stokes theorem, $d \vec{S}$ denotes the infinitesimal areal vector, and $S$ means the area of the Sagnac interferometer.

Note that the relativistic Sagnac effect is dependent on the inner product as $(\vec{\nabla} \times \vec{h}) \cdot \vec{N}_{I}$ for the unit normal vector $\vec{N}_{I}$ to the interferometer plane, whereas the relativistic gyroscope precession by $\vec{h}$ depends mainly on the outer product as $(\vec{\nabla} \times \vec{h}) \times \vec{L}$ for the spin vector $\vec{L}$, roughly speaking.

\section{B. CS modified gravity}

Following Ref. 9], we consider a CS modification to general relativity. The present paper focuses on the leading-order CS correction due to the rotation of a central body. 
Here, the Earth is approximated by a spinning body that is a source of the gravitational field. Therefore, it is sufficient to consider nondynamical CS gravity in this paper, though more dynamical systems such as compact binaries and black hole formations may require a dynamical CS treatment because of their rapid changes in time and space [22].

The exterior weak-field $\vec{h}$ in GR, which causes the LT effect, is known to be [9]

$$
\vec{h}_{L T}=\frac{4 G M R^{2}}{5 c^{3} r^{2}}(\vec{n} \times \vec{\omega}),
$$

where $R$ is the radius of Earth, $M$ is its mass, $\vec{\omega}$ is its angular velocity, $r$ is the distance from the origin, and $\vec{n}$ is the unit vertical vector.

We consider the the CS modified gravity theory by the action as [9]

$$
S=\int d^{4} x \sqrt{-g}\left[-\frac{c^{4}}{16 \pi G} \boldsymbol{R}+\frac{\ell}{12} \theta \boldsymbol{R} \tilde{\boldsymbol{R}}-\frac{1}{2}(\partial \theta)^{2}-V(\theta)+\mathcal{L}_{m a t}\right],
$$

where $\mathcal{L}_{\text {mat }}$ is the Lagrangian density for matter, $g \equiv \operatorname{det}\left(g_{\mu \nu}\right)$, and $\boldsymbol{R}$ is the Ricci scalar, and $\boldsymbol{R} \tilde{\boldsymbol{R}}$ denotes a contraction of the Riemann tensor and its dual, and $\theta$ is a dynamical scalar field with a potential $V(\theta)$. In this theory, we follow Ref. [9] to suppose that the scalar field depends only on cosmic time, $\theta=\theta(t)$, and define $m_{C S} \equiv-3 /\left(\ell \kappa^{2} \dot{\theta}\right)$, where $\kappa=8 \pi G / c^{4}$. The spacetime metric as the weak-field solution to the CS modified field equations appears at the leading order in $g_{0 i}$. It is obtained as [9]

$$
\vec{h}_{C S}=\frac{12 G M}{m_{C S} c^{3} R}\left[C_{1}(r) \vec{\omega}+C_{2}(r) \vec{n} \times \vec{\omega}+C_{3}(r) \vec{n} \times(\vec{n} \times \vec{\omega})\right],
$$

with

$$
\begin{aligned}
& C_{1}(r)=\frac{2 R^{3}}{15 r^{3}}+\frac{2 R}{r} j_{2}\left(m_{C S} R\right) y_{1}\left(m_{C S} r\right), \\
& C_{2}(r)=m_{C S} R j_{2}\left(m_{C S} R\right) y_{1}\left(m_{C S} r\right), \\
& C_{3}(r)=\frac{R^{3}}{5 r^{3}}+m_{C S} R j_{2}\left(m_{C S} R\right) y_{2}\left(m_{C S} r\right),
\end{aligned}
$$

outside the sphere. Here, $j_{\ell}(x)$ and $y_{\ell}(x)$ are spherical Bessel functions of the first and second kind, respectively. Smith et al. obtained both the gravitomagnetic field inside and outside a spinning sphere. We focus on the exterior field, because interferometers are considered in this paper.

Furthermore, curl of the field is obtained for GR as [9]

$$
\left(\vec{\nabla} \times \vec{h}_{L T}\right)=-\frac{4 G M R^{2}}{5 c^{3} r^{3}}[2 \vec{\omega}+3 \vec{n} \times(\vec{n} \times \vec{\omega})],
$$


For the CS term, it becomes [9]

$$
\left(\vec{\nabla} \times \vec{h}_{C S}\right)=-\frac{12 G M}{c^{3} R}\left[D_{1}(r) \vec{\omega}+D_{2}(r) \vec{n} \times \vec{\omega}+D_{3}(r) \vec{n} \times(\vec{n} \times \vec{\omega})\right]
$$

with

$$
\begin{aligned}
& D_{1}(r)=\frac{2 R}{r} j_{2}\left(m_{C S} R\right) y_{1}\left(m_{C S} r\right), \\
& D_{2}(r)=m_{C S} R j_{2}\left(m_{C S} R\right) y_{1}\left(m_{C S} r\right), \\
& D_{3}(r)=m_{C S} R j_{2}\left(m_{C S} R\right) y_{2}\left(m_{C S} r\right) .
\end{aligned}
$$

\section{RELATIVISTIC SAGNAC EFFECT INDUCED BY CS CORRECTION TERMS}

\section{A. Time shift and phase shift}

Substituting Eq. (8) into Eq. (11) leads to the coordinate-time shift as

$$
\begin{aligned}
(c \Delta t)_{L T} & =\frac{8 G M R^{2}}{5 c^{3}} \int_{S}\left[\frac{2 \vec{\omega}+3 \vec{n} \times(\vec{n} \times \vec{\omega})}{r^{3}}\right] \cdot \vec{N}_{I} d S \\
& =\frac{8 G M R^{2} S}{5 c^{3} r^{3}} \vec{N}_{I} \cdot[2 \vec{\omega}-3 \vec{\rho}]
\end{aligned}
$$

where we assumed that the size of the interferometer is much smaller than the radius of the Earth. Similarly, we obtain the time difference due to CS as

$$
\begin{aligned}
(c \Delta t)_{C S} & =\frac{24 G M}{c^{3} R} \int_{S}\left[D_{1}(r) \vec{\omega}+D_{2}(r) \vec{n} \times \vec{\omega}+D_{3}(r) \vec{n} \times(\vec{n} \times \vec{\omega})\right] \cdot \vec{N}_{I} d S \\
& =\frac{24 G M S}{c^{3} R} \vec{N}_{I} \cdot\left[D_{1}(r) \vec{\omega}-D_{2}(r) \vec{\lambda}-D_{3}(r) \vec{\rho}\right]
\end{aligned}
$$

where $\vec{\lambda} \equiv \vec{\omega} \times \vec{n}$ is a vector parallel to a line of latitude on the sphere and $\vec{\rho} \equiv \vec{n} \times(\vec{\omega} \times \vec{n})=$ $\vec{\omega}-(\vec{\omega} \cdot \vec{n}) \vec{n}$ is a vector parallel to a line of longitude on the sphere.

The order of magnitude of time difference by LT effects in GR is

$$
(c \Delta t)_{L T} \sim \frac{8 G M S \omega}{5 c^{3} R} .
$$

The magnitude of Eq. (14) is roughly

$$
(c \Delta t)_{C S} \sim \frac{24 G M S \omega}{c^{3} m_{C S} R^{2}} .
$$


From these equations, one can estimate the size of the relativistic Sagnac effect due to LT and CS, respectively.

The altitudinal dependence of CS effects might be negligible very near the surface of Earth. However, the difference between the ground-based interferometer and one at an altitude $\sim 400 \mathrm{~km}$ (corresponding to the space station) might become of the order of the unity for $m_{C S} \sim 0.01-0.001 \mathrm{~km}^{-1}$, for instance. This point will be discussed later in more detail.

Here, we mention experimantal realities. With $m_{C S}=0.001 \mathrm{~km}^{-1}$, one finds the shift $c \Delta t$ is of the order $10^{-18} \mathrm{~km}$ for an interferometer with an area of a square kilometer. In terms of strain $c \Delta t / L$, where $L$ is the size of the interferometer, the strain is $\sim 10^{-18}$. For a more modest meter-scale interferometer, the strain is three orders of magnitude smaller. While the modest setup might be more promising, the strain of $10^{-21}$ is apparently reachable by gravitational wave detecters such as LIGO (on the ground) and LISA (in the space). However, this is not the case. The strain under study is essentially a DC (namely zerofrequency) strain, while gravitational-wave interferometers search high-frequency signals and they try to kill various noises at low frequency.

\section{B. Numerical Calculations}

According to previous works on the precession, [7 9, 12] there has been a constraint on $m_{C S}$ as $m_{C S}>0.001\left[\mathrm{~km}^{-1}\right]$, roughly speaking. Taking account of this existing constraint, numerical calculations are done for a parameter region $0.001\left[\mathrm{~km}^{-1}\right]<m_{C S}<0.1\left[\mathrm{~km}^{-1}\right]$ in this paper. The time shift $(c \Delta t)_{C S}$ depends on four parameters as the CS mass parameter $m_{C S}$, the interferometer direction $\alpha$, the latitude $\phi$, and the altitude $h$, where $\alpha$ is defined as a horizontal angle measured clockwise from a north base line or meridian. For instance, $\alpha=0^{\circ}$ and $90^{\circ}$ correspond to the direction along $\vec{\rho}$ and $\vec{\lambda}$, respectively. Furthermore, the shift is dependent also on the zenith angle, which is not considered in the present paper.

As a reference, let us consider the time shift $(c \Delta t)_{L T}$ due to LT effects in GR. It is useful to define $\Delta_{L T}$ as $(c \Delta t)_{L T}$ in the units of $8 G M S \omega / 5 c^{3} R$, namely the angular part of $(c \Delta t)_{L T}$ as $\vec{N}_{I} \cdot[2 \vec{\omega}-3 \vec{\rho}] /|\vec{\omega}|$. Figure 1 shows the dependence of $\Delta_{L T}$ on the latitude and the direction. It follows that there are no oscillating behaviors in LT effects. Note that LT effects vanish in the interferometer direction as $\alpha=90^{\circ}$ and $270^{\circ}$ almost everywhere except 
for polar regions. This suggests that the eastbound direction of the interferometer might be preferred for testing CS, separately, because LT effects on this interferometer cancel out.

First, we consider ground-based experiments $(h=0)$, for which $(c \Delta t)_{C S}$ depends on the other three parameters $m_{C S}, \alpha$ and $\phi$. It is useful to define $\Delta_{C S}$ as $(c \Delta t)_{C S}$ in the units of $24 G M S \omega / c^{3} R$, namely the angular part of $(c \Delta t)_{C S}$. Figure 1 shows numerical calculations of the dependence of the time shift $\Delta_{C S}$ on the latitude $\phi$ and the direction $\alpha$, where we assume $m_{C S}=0.001\left[\mathrm{~km}^{-1}\right]$ and the vertical axis denotes $\Delta_{C S}$. For this case, the CS effect becomes the largest around $\alpha=130^{\circ}$ and $300^{\circ}$ for wide latitude regions from the equator to the middle latitude except for the polar regions.

The latitudinal and directional dependence is weak around $m_{C S} \sim 0.1$, while it is strong around $m_{C S} \sim 0.001-0.01$. Therefore, one can say that the CS latitudinal and directional effects might be important in experiments, especially when we investigate the parameter region $m_{C S} \sim 0.001-0.01$. Even if any imprint by CS were marginally detected in the future (presumably at a low signal-to-noise ratio), it would be difficult to disentangle the CS signal from other effects without taking account of these dependencies. A comparison of phase measurements at two (or more) directions at different latitudes would be helpful for improving the CS bound or distinguishing the CS signal from others. Namely, a signalto-noise ratio could be enhanced by a combined analysis of phase measurements at different latitudes and directions.

Before closing this section, we mention the altitudinal dependence of the CS effect on the Sagnac interference. In order to understand the dependence, let us suppose two interferometers at different altitudes: One is located at $R$ and the other is at $R+h$. Eq. (14) suggests that a height difference $h$ makes a change in the time difference, where we assume the same interferometers. The relative difference between two measurements is of the order of $\sim m_{C S} h$. For instance,

$$
\begin{aligned}
\left|\frac{(c \Delta t)_{R+h}-(c \Delta t)_{R}}{(c \Delta t)_{R}}\right|_{C S} & \sim \frac{h \times \frac{\partial}{\partial r}(c \Delta t)_{R}}{(c \Delta t)_{R}} \\
& \sim 0.002\left(\frac{m_{C S}}{0.001 \mathrm{~km}^{-1}}\right)\left(\frac{h}{1600 \mathrm{~m}}\right),
\end{aligned}
$$

where Eq. (14) is used and we assume Denver as a high city. Hence, the height difference of the CS effect is very small on the surface of the Earth. If the Sagnac interferometer were 
located in the space, however, the altitudinal difference might become significant as

$$
\left|\frac{(c \Delta t)_{R+h}-(c \Delta t)_{R}}{(c \Delta t)_{R}}\right|_{C S} \sim 0.4\left(\frac{m_{C S}}{0.001 \mathrm{~km}^{-1}}\right)\left(\frac{h}{400 \mathrm{~km}}\right),
$$

where we assumed the international space station (ISS) as an example. Figure 2 shows a comparison between the ground level and the ISS site regarding the oscillating behaviors in terms of $m_{C S}$. The altitudinal effect might make a more complicated form of oscillating behaviors. Such a altitudinal dependence might be helpful for a future test. Figure 3 shows the time shift as a function of the orbital phase angle $\theta$, which is nearly proportional to time because the ISS moves on a nearly polar orbit. The ISS is orbiting around the Earth with the period of nearly 90 minutes. Hence, the apparent zero-frequency strain due to the gravitomagnetic effects on such a satellite experiment can vary with time. This time variability might help separate the effects from the others. One could use this altitudinal dependence in order to place tighter constraints on the mass parameter in the future. For instance, a commercial ring laser as a long-term stable gyroscope is used in a number of airplanes. Such an instrument, if it is sufficiently improved in the future, may be used for the present purpose.

\section{CONCLUSION}

In this paper, we have investigated relativistic Sagnac effects in CS modified gravity. The altitudinal, latitudinal and directional dependence of relativistic Sagnac effect in the CS model is oscillatory in terms of the CS parameter $m_{C S}$.

We have compared the CS effects on Sagnac interferometers with the general relativistic Lense-Thirring (LT) effects. LT effects on the eastbound interferometer cancel out. Therefore, our numerical calculations have suggested that the eastbound Sagnac interferometer might be preferred for testing CS separately.

For some region of the CS parameter $m_{C S} \sim 0.01-0.001\left[\mathrm{~km}^{-1}\right]$, the possible altitudinal dependence might become important when we consider a space experiment such as the ISS site at $h \sim 400 \mathrm{~km}$. Further investigations along this course might be interesting as a future work.

We would like to thank N. Yunes, S. Takeuchi and K. Edamatsu for useful discussions. We wish to thank the referee for useful comments on experimental realities and instabilities 
of nondynamical CS models. This work was supported in part by JSPS Grant-in-Aid for JSPS Fellows, No. 24108 (K.Y.), and JSPS Grant-in-Aid for Scientific Research (Kiban C), No. 26400262 (H.A.).

[1] J. Polchinski, Superstring Theory and Beyond String Theory vol.2 (Cambridge University Press, Cambridge, UK, 1998).

[2] A. Ashtekar, A.P. Balachandran, and S. Jo, Int. J. Mod. Phys. A 4, 1493 (1989).

[3] V. Taveras and N. Yunes, Phys. Rev. D 78, 064070 (2008); S. Mercuri and V. Taveras, Phys. Rev. D 80, 104007 (2009).

[4] S. Deser, R. Jackiw, and S. Templeton, Ann. Phys. (N.Y.) 140, 372 (1982); 185, 406(E) (1988); 281, 409 (2000).

[5] B. A. Campbell, M. J. Duncan, N. Kaloper, and Olive, Phys. Lett. B 251, 34 (1990); Nucl. Phys. B351, 778 (1991).

[6] S. Alexander and N. Yunes, Phys. Rep. 480, 1 (2009).

[7] S. Alexander and N. Yunes, Phys. Rev. Lett. 99, 241101 (2007).

[8] S. Alexander and N. Yunes, Phys. Rev. D 75, 124022 (2007).

[9] T. L. Smith, A. L. Erickcek, R. R. Caldwell, and M. Kamionkowski, Phys. Rev. D 77, 024015 (2008).

[10] Y. Ali-Haimoud and Y. Chen, Phys. Rev. D 84, 124033 (2011).

[11] N. Yunes, and D.N. Spergel, Phys. Rev. D 80, 042004 (2009).

[12] Y. Ali-Haimoud, Phys. Rev. D 83, 124050 (2011).

[13] M. Zych, F. Costa, I. Pikovski, C. Brukner, Nature Communications 2, 505 (2011).

[14] H. Okawara, K. Yamada, H. Asada, Phys. Rev. Lett. 109, 231101 (2012).

[15] H. Okawara, K. Yamada, H. Asada, Phys. Rev. D 87, 084038 (2013).

[16] G. E. Stedman, Rep. Prog. Phys. 60, 615 (1997).

[17] K. U. Schreiber, and J. R. Wells, Rev. Sci. Instrum. 84, 041101 (2013); (http://dx.doi.org/10.1063/1.4798216).

[18] A. Tartaglia, Journal of Physics: Conference Series, 453, 012019 (2013) (arXiv:1212.2880).

[19] J. Anandan, Phys. Rev. D 15, 1448 (1977).

[20] G. Rizzi, and M. L. Ruggiero, Gen. Rel. Gravit. 35, 2129 (2003). 
[21] P. Maraner, and J. Zendri, Gen. Rel. Gravit. 441713 (2012).

[22] Note that the nondynamical CS theory suffers from a rather serious instability [23] and hence it is not clear how viable this alternative theory is. In the future, the CS model adopted in the present paper might be replaced with a more improved one.

[23] S. Dyda, E. E. Flanagan and M. Kamionkowski, Phys. Rev. D 86, 124031 (2012). 

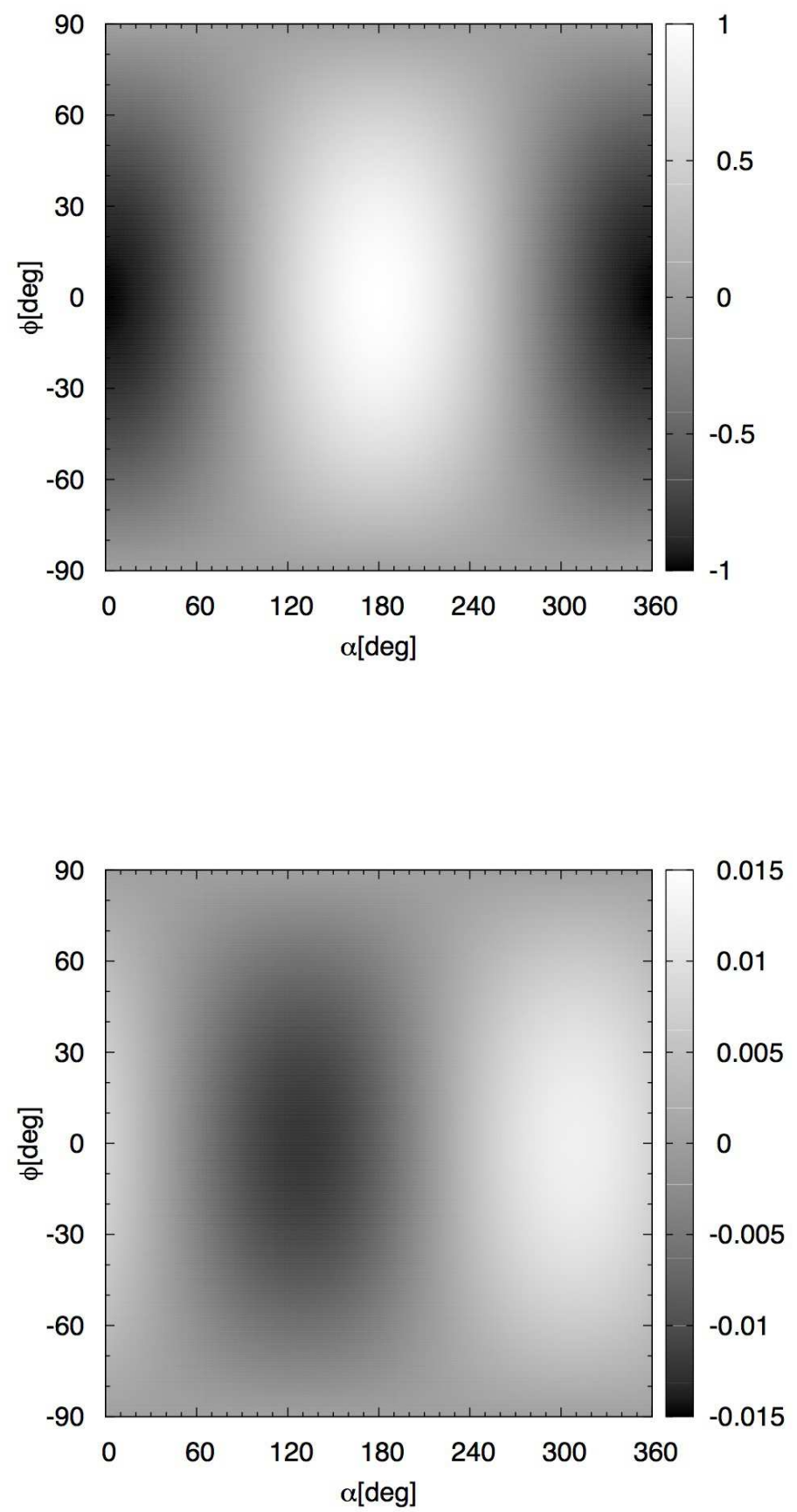

FIG. 1: Contour maps for the dependence of time shift on the interferometer direction angle $\alpha$ and the latitude $\phi$, where the height corresponds to the angular part of $(c \Delta t)$. Top: $\Delta_{L T}$ by LT effects in GR. Bottom: $\Delta_{C S}$ by CS effects. 


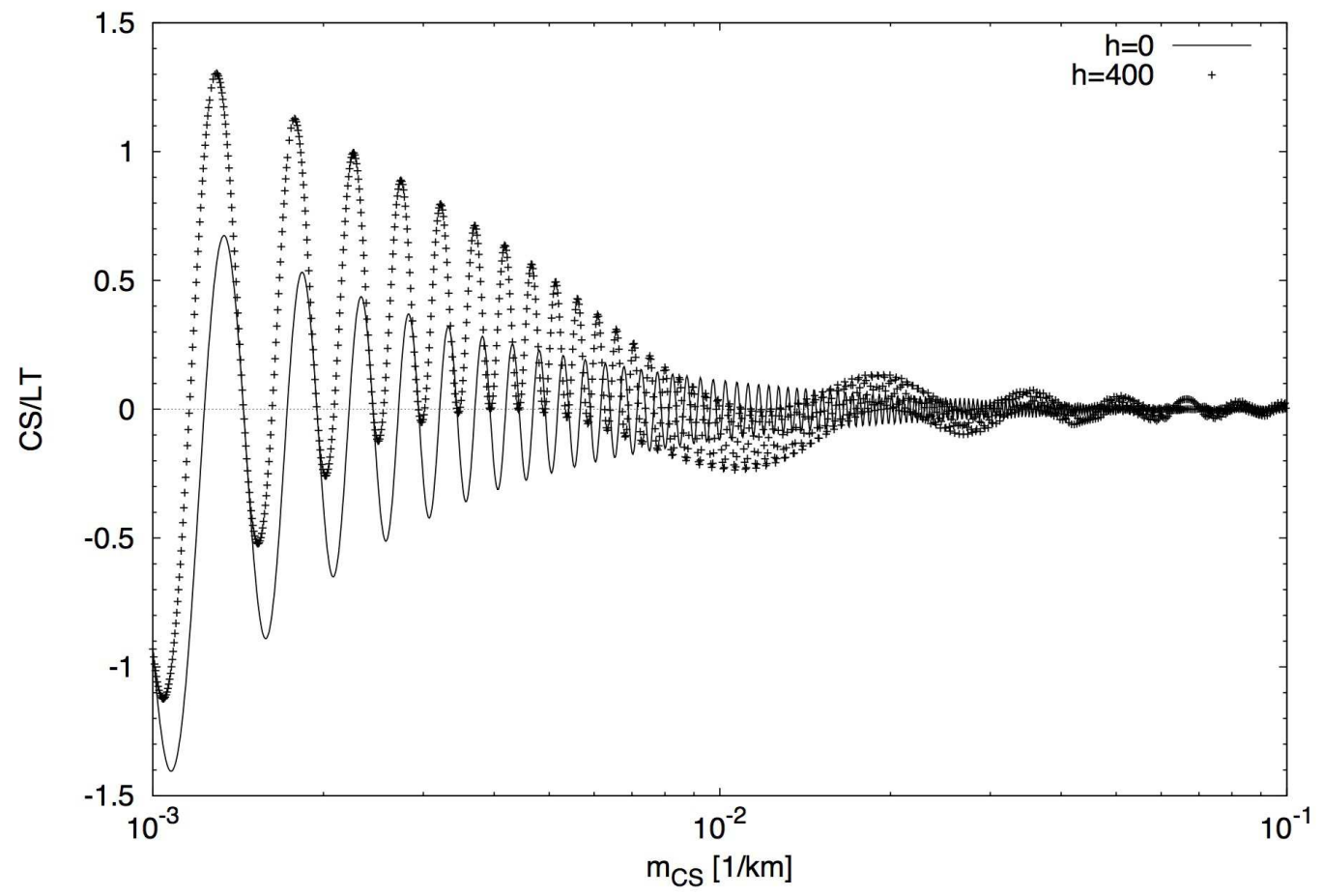

FIG. 2: The ratio of $(c \Delta t)_{C S} /(c \Delta t)_{L T}$ at the ground level and the ISS site at $h \sim 400 \mathrm{~km}$. For its simplicity, we assume the equatorial case as $\phi=0^{\circ}$ and the northbound direction as $\alpha=0^{\circ}$. This figure suggests that the altitudinal effect might make a more complicated form of oscillating behavior in terms of $m_{C S}$ compared with the ground level. 


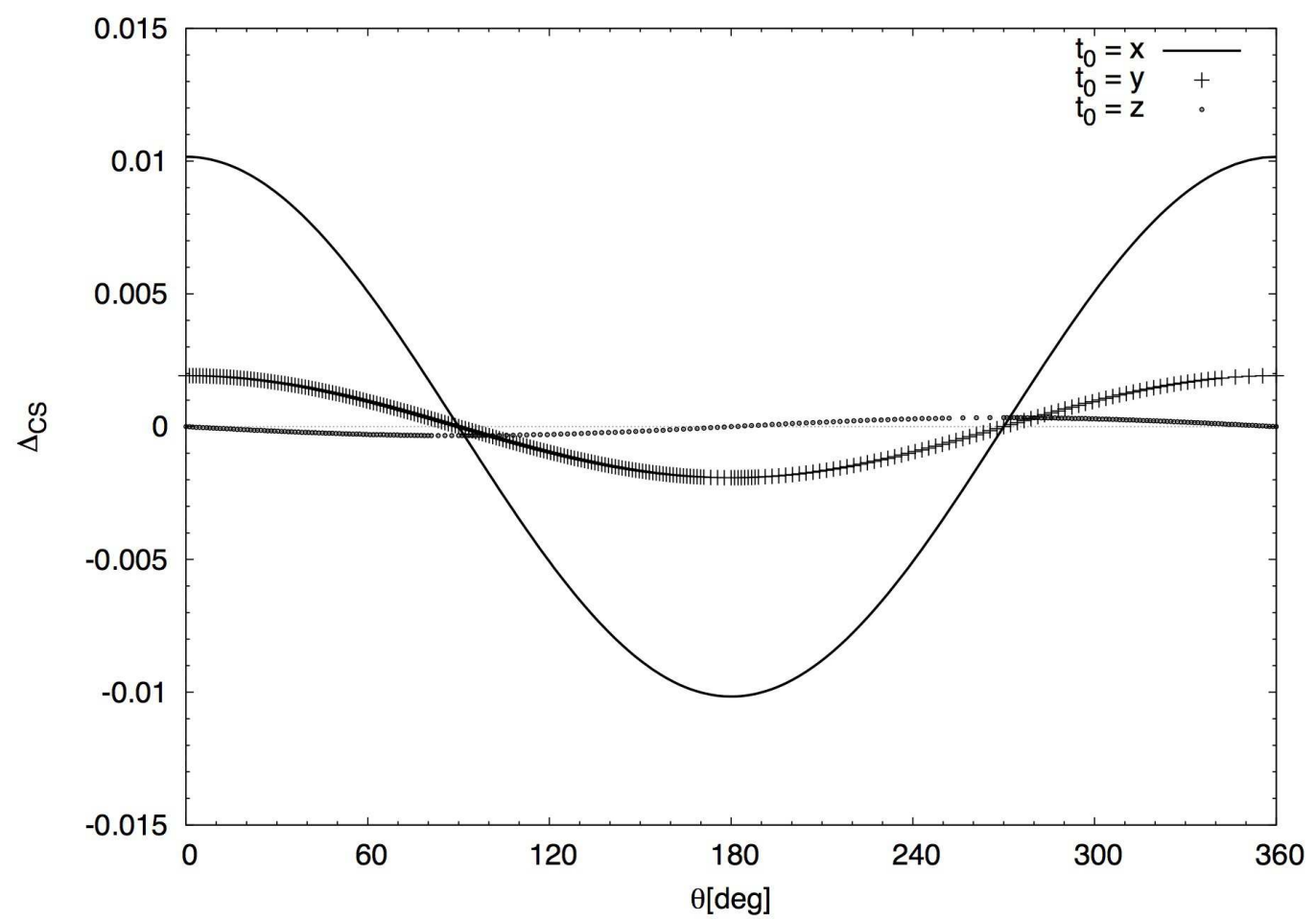

FIG. 3: Time shift as a function of the orbital phase angle $\theta$ that is measured from the initial direction on the equator. For its simplicity, we assume the corotation of the ISS in a polar orbit around the Earth $(h \sim 400 \mathrm{~km})$. For the circular orbit, $\theta$ is proportional to time, where $\theta=$ $0^{\circ}, 90^{\circ}, 180^{\circ}$ and $270^{\circ}$ are corresponding to the passage time of the equatorial plane, a pole of Earth, the equatorial plane and the opposite pole, respectively. We consider three directions of the interferometer: The labels as $\mathrm{x}, \mathrm{y}$ and $\mathrm{z}$ denote the eastbound direction, northbound one and vertical one, respectively, at the initial time as $\theta=0^{\circ}$. 